\title{
PATRONES ALIMENTARIOS EN EMBARAZADAS DE BAJO PESO DE LA REGIÓN METROPOLITANA
}

\section{FEEDING PATTERNS OF UNDERWEIGHT PREGNANT WOMEN FROM THE METROPOLITAN REGION}

\author{
Oscar Castillo V. (1), Francisco Mardones S. (2), Jaime Rozowski N. (1)
}

(1) Unidad de Intervención Nutricional, Departamento de Nutrición, Diabetes y Metabolismo, Facultad de Medicina, Pontificia Universidad Católica de Chile. Santiago, Chile.

(2) Departamento de Salud Pública,

Facultad de Medicina, Pontificia Universidad Católica de Chile. Santiago, Chile.

\begin{abstract}
The aim of this study was to determine food and nutrient intake of underweight pregnant women of the SouthEast Metropolitan Region. We studied 411 pregnant women attending health control clinics in years 2002 to 2004. Dietary intake was determined by a 24-hour recall taken at home in week 20 and 35 by trained nutritionists. Nutrient intake was analyzed using the software Food Processor 7.9, and compared with the recommended servings from the Ministry of Health. Baseline characteristics (mean $\pm S D$ ): age $23.8 \pm 5.4$ years, weight $49.3 \pm 4.7 \mathrm{~kg}$, height $156.9 \pm 5.9 \mathrm{~cm}$, gestational age $11.5 \pm 3$, 7 weeks. Compared with the recommendations the intake of energy and macronutrients was adequate while the intake of vitamin $A, C, E$ and B6, calcium and zinc were $75 \%$ of the recommendations. The intake of omega-3 fatty acids averaged $61 \pm 50$ and $71 \pm 24 \mathrm{mg} /$ day in week 20 and 35 , respectively, a sixth of the NIH recommendation. Deficient intakes were found for dairy products, fruits, vegetables and meats, however there was a higher intake of cereals. Conclusions: The nutrient intake of pregnant women in this group is insufficient in several micronutrients, resulting from inadequate food intake; this should be corrected by improving the quality of their diet or other measures such as supplementation with these micronutrients. Key words: feeding, pregnancy, nutritional status.
\end{abstract}

Este trabajo fue recibido el 5 de Diciembre de 2010 y aceptado para ser publicado el 19 de Mayo de 2011.

\section{INTRODUCCIÓN}

Está bien establecido que una buena nutrición materna es un factor clave en la prevención de morbilidad y mortalidad infantiles y que durante el embarazo se produce un incremento en los requerimientos de nutrientes (1).

Una proporción sustancial de mujeres en países desarrollados y subdesarrollados consumen dietas que contienen menos que la cantidad recomendada de ciertos micronutrientes, las cuales han sido asociadas con el curso y los resultados del embarazo (2,3). La mayoría de esos déficits en la dieta son para zinc, folatos, hierro y calcio. Estas carencias varían de acuerdo al patrón alimentario; es decir las características alimentarias comunes a un grupo de individuos con características similares desde el punto de vista sociocultural.
Determinar el consumo de alimentos y de nutrientes de un grupo, es una tarea difícil de conseguir por diversos factores, entre los que se cuentan no contar con un método exacto y fácil de realizar. Sin embargo, dada la importancia de saber el modo en que la población se alimenta, es que se utilizan métodos que no son lo suficientemente acuciosos en determinarlo (4).

Dentro de las encuestas alimentarias encontramos la encuesta por recordatorio de 24 horas. Esta tiene por objetivo conocer el consumo de alimentos del día anterior de la persona entrevistada, incluyendo no solo las preparaciones consumidas si no que también cada ingrediente que la compone, proporcionando datos sobre la ingesta habitual de alimentos. Permite estudiar la relación entre dieta y enfermedad, no se modifican los patrones alimentarios de los entrevistados y el encues- 
tado no necesariamente debe saber leer y escribir (5).

El objetivo de este estudio fue determinar el patrón alimentario en un grupo de embarazadas de bajo peso en la semana 20 y 35 de su embarazo, para conocer las principales carencias además de comparar las ingestas en ambas etapas del embarazo.

\section{SUJETOS Y MÉTODO}

La población de estudio correspondió a 411 (de un total de 506) embarazadas con bajo peso pertenecientes al grupo control de un proyecto mayor, de intervención alimentaria con una leche rica en micronutrientes (MAMAN), en embarazadas bajo peso del sector Sur-Oriente de la Región Metropolitana, sus características han sido descritas previamente. (6)

Las mujeres debían tener 18 o más años, con una paridad entre 0 y 5 hijos, presentar un peso para la talla por debajo de los límites normales según la curva de peso para talla en uso por el Ministerio de Salud (7) y contar con 20 semanas y menos de duración del embarazo.

El grupo control, que recibió el producto Purita Fortificada (PF), fue controlado en los consultorios de acuerdo a los procedimientos existentes, que consisten en controles mensuales. El aporte de PF consiste en 2 $\mathrm{Kg}$. de leche entera por mes (26\% de materia grasa). Estas madres de bajo peso reciben además $2 \mathrm{~kg}$. de arroz por mes.

Del total de embarazadas randomizadas al grupo PF (560), 411 se incluyeron en este análisis, ya que estas son las embarazadas que cuentan con encuestas alimentarias tanto en la semana de gestación 20 como en la 35 .

En este estudio se utilizó 1 encuesta por recordatorio de 24 horas realizada en el domicilio. A pesar de que sólo se realizó una encuesta alimentaria en cada tiempo, la ventaja de que sea realizado en el hogar, es que se puede observar directamente las medidas caseras y los alimentos utilizados por la embarazada, dando mayor precisión en la determinación de la cantidad y calidad de alimentos consumido por esta.

Los alimentos y preparaciones, registradas en medidas caseras en las encuestas, luego de ser aplicadas, fueron transformados a gramos o $\mathrm{ml}$ por medio de tablas especialmente diseñadas para esto (8) y posteriormente analizadas por medio del software The Food Processor 2 (versión 7,9). Este último a pesar de tratarse de un programa de análisis de alimentos extranjero, preferimos usarlo por la gran cantidad de datos que aporta. Este programa ha sido utilizado anteriormente por nuestro grupo (9-11).

Se analizaron ambas encuestas por separado (semana 20 y 35), las comparaciones se realizaron pareando las encuestas de cada una de las embarazadas estudiadas.

\section{ANÁLISIS ESTADÍSTICO}

Se presentan las variables numéricas como promedio \pm desviación estándar. Las variables categóricas se presentan como número de casos y porcentajes.

Para comparar entre semanas 20 y 35 se usó test $\mathrm{t}$ de Student para muestras pareadas. El porcentaje de adecuación de calorías y nutrientes en los individuos muestrales se estableció comparándolos con recomendaciones internacionales.

Para la aplicación de algunos test estadísticos se determinó la normalidad de las variables de interés mediante el test de Kolmogorov-Smirnov. Cuando los tamaños muestrales fueron grandes, se asumió normalidad de los promedios por el Teorema Central del Límite.

El estudio fue aprobado por el Comité de Etica de la Pontificia Universidad Católica de Chile

\section{RESULTADOS}

La tabla 1 muestra las características generales de la población en estudio al reclutamiento. Las embarazadas tenían un promedio de edad cercano a los 24 años con 11 semanas de gestación en promedio. El índice de masa corporal estaba bajo los límites inferiores de normalidad según la curva de evaluación Rosso-Mardones, por lo que todas ellas tenían un estado nutricional deficiente al momento del ingreso.

La tabla 2 resume la ingesta de energía y macronutrientes para el grupo de estudio en la semana 20 y 35 de gestación. Al igual que en la población general, el mayor aporte de las calorías viene de los carbohidratos, seguidos por las grasas y finalmente las proteínas. La distribución de los diferentes tipos de grasas observados en este grupo, muestra un consumo similar de cada uno de ellos. El consumo de colesterol está dentro de las recomendaciones y la razón omega 3/omega 6 es alta, por sobre lo recomendado (12).

La ingesta de micronutrientes se muestra en la tabla 3 , se seleccionaron estos en particular dado que son los que habitualmente presentan deficiencias en su consumo en las diferentes poblaciones estudiadas tanto en Chile como otras partes del mundo (13-20). El comportamiento observado en este grupo es similar a los descritos en estos estudios.

Las adecuaciones del consumo de energía, proteínas y micronutrientes seleccionados respecto de las recomendaciones internacionales (21-25) para este grupo de estudio, las observamos en la tabla 4 , figura 1 y 2 ; se aprecian deficiencias importantes en la adecuación de consumo de micronutrientes, no así para los macronutrientes, como ha sido mostrado anteriormente en otros estudios realizados en otras poblaciones del país (9).

La tabla 5 muestra el consumo de alimentos de este 
grupo de embarazadas expresado en gramos por día tanto en la semana 20 como en la semana 35, llama la atención el alto consumo de cereales y el bajo consumo de lácteos de este grupo.

En la tabla 6 observamos las porciones de los diferentes grupos de alimentos consumidos por estas embarazadas y las adecuaciones del consumo de estas porciones de alimentos de acuerdo a las recomendaciones del Ministerio de Salud para las embarazadas de nuestro país (26). Se hace evidente el consumo excesivo del grupo de cereales y llama la atención el déficit de consumo de todo el resto de los grupos de alimentos con adecuaciones en general cercanas al $50 \%$ de lo recomendado.

En la tabla 7 se comparan las ingestas del grupo estudiado en la semana 20 versus la semana 35 , las diferencias encontradas fueron un mayor consumo de proteínas en la semana 20 respecto de la semana 35 , además de un mayor consumo de magnesio y zinc en la semana 20 respecto de la semana 35 , resultados que son coincidentes con las diferencias en el consumo de alimentos.

\section{DISCUSIÓN}

En nuestro país no ha existido información sobre la dieta de la población desde la década del 60 (27),

\section{TABLA 1}

\section{Características generales de la muestra $(n=411) *$}

Edad al ingreso (años)

Edad gestacional al ingreso (semanas)

Peso al inicio del embarazo (kilos)

Talla al ingreso (centímetros)

Índice peso/talla al ingreso

Índice de masa corporal $\left(\mathrm{k} / \mathrm{m}^{2}\right)$ al ingreso

$$
\begin{gathered}
23,8 \pm 5,4 \\
11,5 \pm 3,7 \\
49,3 \pm 4,7 \\
156,9 \pm 5,9 \\
91,1 \pm 5,5 \\
20,2 \pm 1,2
\end{gathered}
$$

* Promedio \pm DE

TABLA 2

Ingesta de energía y macronutrientes en semana 20 y 35 (n=411)

$\begin{array}{lccc} & \text { Semana 20* } & \text { \% VCT^ } & \text { Semana 35* VCT^ } \\ \text { Energía (Kcal./día) } & 2267 \pm 696 & & 2202 \pm 696 \\ \text { Proteínas (g/día) } & 82,5 \pm 28,5 & 14,5 & 77,9 \pm 26,5 \\ \text { Carbohidratos (g/día) } & 345,5 \pm 114,7 & 60,9 & 338,5 \pm 123,8 \\ \text { Grasas totales (g/día) } & 65,2 \pm 26,3 & 25,8 & 62,2 \pm 24,9 \\ \text { Grasas saturadas (g/día) } & 15,7 \pm 9,1 & 6,2 & 14,5 \pm 8,7 \\ \text { Grasas mono (g/día) } & 18,1 \pm 8,5 & 7,2 & 17,2 \pm 8,5 \\ \text { Grasas poli (g/día) } & 17,2 \pm 9,3 & 6,8 & 16,9 \pm 8,5 \\ \text { Grasas omega 3 (g/día) } & 0,61 \pm 0,5 & & 0,71 \pm 2,4 \\ \text { Grasas omega 6 (g/día) } & 16,6 \pm 9,1 & & 16,3 \pm 8,2 \\ \text { Razón omega 6/omega 3 } & 27,2 & & 22,9 \\ \text { Colesterol (mg/día) } & 185,4 \pm 170,1 & & 180,3 \pm 184,8 \\ \text { Fibra (g/día) } & 26,5 \pm 13,6 & & 24,7 \pm 12,0\end{array}$


complementada posteriormente con la encuesta ECEN de 1974 (28) y sólo esfuerzos aislados de algunos investigadores nos han dado luces de las deficiencias y excesos de pequeños grupos de nuestra población $(9,10,13,18,29)$.

Las embarazadas tienen características diferentes a la población general desde el punto de vista de sus requerimientos energéticos y de micronutrientes (2125,31 ), siendo mayores respecto de la población general, es por este motivo que se convierte en un grupo más susceptible de presentar problemas relacionados con el déficit de consumo de macro y micronutrientes.

Múltiples complicaciones derivadas de la mala alimentación han sido descritos en este grupo de estudio, entre los más comunes están la anemia, preeclampsia, eclampsia, mayor tasa de abortos espontáneos y desmineralización ósea, entre otros, desde el punto de vista de la madre y bajo peso de nacimiento, defectos del desarrollo del tubo neural, menor talla de nacimiento, infecciones perinatales, entre otros, por parte del recién nacido (31-46).
Nuestro grupo de estudio mostró ingestas de energía y macronutrientes en promedio adecuados para su estado fisiológico, lo que se observa en la mayoría de los estudios realizados tanto en el extranjero como en nuestro país. Un estudio realizado en Concepción (13), utilizando dos encuestas por recordatorio de 24 horas, observó que la adecuación de energía de 243 embarazadas estudiadas fue de $117 \%$, además de presentar un $153 \%$ de adecuación para el consumo de proteínas. Por otra parte Pobocik y colaboradores (16) en una muestra de 434 embarazadas de Guam mostró una adecuación de $99 \%$ en energía y $166 \%$ en proteínas al realizar una encuesta por recordatorio de 24 horas, la misma situación ha sido descrita en embarazadas estudiadas en España (19), Perú (20), Venezuela (15) y Finlandia (18). Estos resultados sugieren que en general el consumo de energía y proteínas está asegurado en la mayoría de los países, por tanto no parece ser el tema de mayor preocupación en estos momentos.

El consumo de grasas de las embarazadas estudiadas al igual que el consumo de colesterol se encuentra dentro

TABLA 3

Ingesta de micronutrientes seleccionados $(n=411) *$

\begin{tabular}{lcc} 
& Semana 20 & Semana 35 \\
\hline Vitamina A ( $\mu$ g/día) & $971,0 \pm 1637,9$ & $940,5 \pm 1420,9$ \\
Vitamina C (mg/día) & $115,7 \pm 75,4$ & $116,0 \pm 92,7$ \\
Vitamina E (mg/día) & $20,5 \pm 10,9$ & $20,8 \pm 11,5$ \\
Vitamina B6 (mg/día) & $1,7 \pm 0,8$ & $1,6 \pm 0,8$ \\
Calcio (mg/día) & $933,1 \pm 420,0$ & $887,4 \pm 389,9$ \\
Hierro (mg/día) & $19,6 \pm 7,7$ & $19,6 \pm 11,6$ \\
Magnesio (mg/día) & $272,3 \pm 110,5$ & $254,4 \pm 92,7$ \\
Zinc (mg/día) & $10,8 \pm 4,0$ & $10,1 \pm 3,8$ \\
\hline * Promedio \pm DE & & \\
\hline
\end{tabular}

TABLA 4

Adecuación del consumo energético y de proteínas (n=411)

\begin{tabular}{|lcccc|} 
& Semana 20 & \% Adecuación & Semana 35 & \% Adecuación \\
\hline Energía (Kcal./día) & $2267 \pm 696$ & 104,2 & $2202 \pm 696$ & 101,2 \\
Proteínas (g/día) & $82,5 \pm 28,5$ & 137,5 & $77,9 \pm 26,5$ & 129,8 \\
\hline Ref: 21 & & &
\end{tabular}



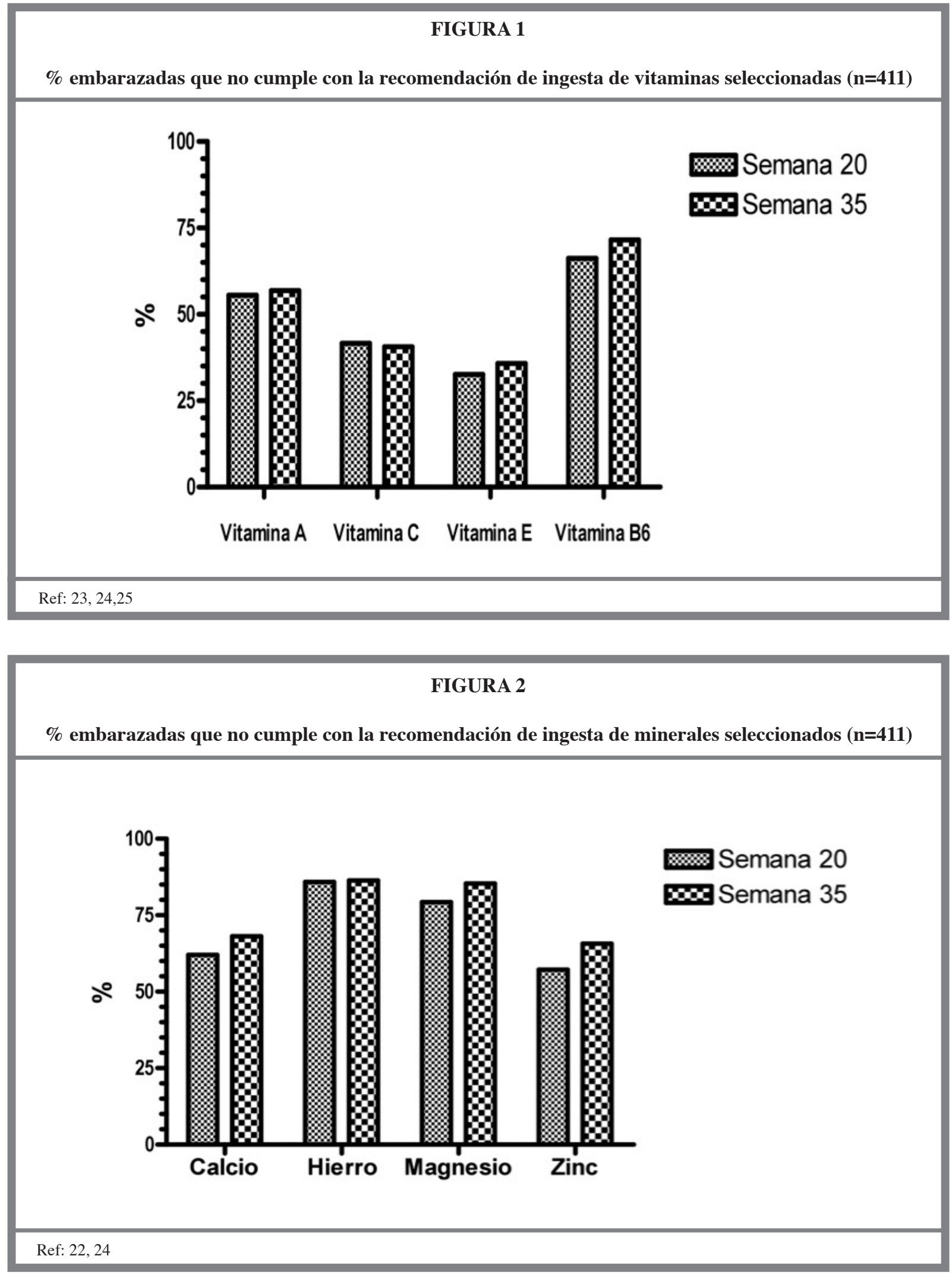
de las recomendaciones (26), tal como ha sido descrito anteriormente en la población chilena, la razón de ácidos grasos omega6/omega 3 es muy alta, bastante lejana a lo recomendado $(9,12)$.

Sabiendo que el consumo de energía y macronutrientes es cubierto por las embarazadas la atención se centra en el consumo de micronutrientes. El grupo estudiado en promedio cubre sus requerimientos de vita- minas, sin embargo al realizar un estudio mas detallado, persona a persona, se aprecia que alrededor de la mitad de las mujeres no alcanza a cubrir sus requerimientos de vitamina A, un $40 \%$ no cubre sus requerimientos de vitamina $\mathrm{C}$, un $35 \%$ no alcanza sus requerimientos de vitamina $\mathrm{E}$ y alrededor de un $70 \%$ no lo hace para vitamina B6 en ambos períodos de estudio. Un estudio realizado en Temuco (14), demostró que el 23,4\% de las

\section{TABLA 5}

\section{Consumo de alimentos (ml ó g/día) $(\mathrm{n}=411)^{*}$}

\begin{tabular}{lcc}
\hline Grupo Alimentos & Semana 20 & Semana 35 \\
\hline Lácteos & $589,3 \pm 382,1$ & $610,2 \pm 464,5$ \\
Verduras & $176,8 \pm 138,6$ & $165,3 \pm 119,5$ \\
Frutas & $306,5 \pm 283,5$ & $289,7 \pm 286,1$ \\
Carnes rojas & $64,4 \pm 70,2$ & $58,4 \pm 59,7$ \\
Carnes blancas & $17,3 \pm 43,5$ & $17,3 \pm 38,1$ \\
Pescado y mariscos & $10,0 \pm 43,3$ & $8,7 \pm 34,3$ \\
Pan & $255,8 \pm 129,6$ & $244,4 \pm 123,7$ \\
Papas & $92,8 \pm 135,6$ & $87,0 \pm 122,4$ \\
Cereales & $80,5 \pm 77,8$ & $83,1 \pm 75,1$ \\
Legumbres & $13,0 \pm 37,1$ & $11,1 \pm 33,2$ \\
Huevos & $18,8 \pm 36,3$ & $17,9 \pm 39,3$ \\
Aceite & $16,6 \pm 12,5$ & $16,1 \pm 11,6$ \\
Grasas & $16,5 \pm 24,3$ & $16,9 \pm 26,9$ \\
Azúcar & $22,2 \pm 15,9$ & $21,6 \pm 15,5$ \\
Bebidas azucaradas & $136,5 \pm 182,4$ & $124,6 \pm 177,0$
\end{tabular}

TABLA 6

Consumo y adecuación de alimentos (porciones/día) $(\mathrm{n}=411) *$

\begin{tabular}{|lcccc}
\hline Grupo Alimentos & Semana 20 & Adecuación & Semana 35 & Adecuación \\
\hline Cereales & 9,5 & $190 \%$ & 9,2 & $184 \%$ \\
Verduras & 2,2 & $55,5 \%$ & 2,1 & $52,5 \%$ \\
Frutas & 2,5 & $62,5 \%$ & 2,4 & $60 \%$ \\
Lácteos & 2,9 & $58 \%$ & 3,1 & $62 \%$ \\
Carnes & 1,7 & $56,7 \%$ & 1,4 & $46,7 \%$ \\
Grasas y aceites & 1,6 & $80 \%$ & 1,6 & $80 \%$ \\
\hline * Ministerio de Salud & & & &
\end{tabular}


embarazadas no cubría un $75 \%$ de sus requerimientos de vitamina C. Los estudios realizados en el extranjero no muestran una realidad muy diferente, el estudio de Guam (16), muestra que las embarazadas estudiadas cubrían un $49 \%$ de sus requerimientos de ácido fólico y un $57 \%$ de vitamina E. Por su parte, un estudio realizado en Nigeria (17), señala que un $33 \%$ de las mujeres alcanzaba los requerimientos de ácido fólico. En España, la muestra de 493 embarazadas a las que se realizó una encuesta de 24 horas (19) solo un 42,3\% de las embarazadas estudiadas alcanzaba los requerimientos de ácido fólico. En Latinoamérica, coincidentemente un $20 \%$ de las embarazadas de Perú (20) y Venezuela (15) cubría sus requerimientos de ácido fólico.

En cuanto al consumo de minerales, las embarazadas estudiadas cubrían un 62 y $68 \%$ de sus requerimientos de calcio en la semana 20 y 35 respectivamente, el hierro y magnesio presentaba adecuaciones cercanas al $80 \%$ en ambos períodos de medición y el consumo de zinc bordeaba el $60 \%$ de la adecuación. Los datos encontrados en este estudio son coincidentes con la mayoría de los estudios realizados en el tema tanto en el país como en el extranjero. El estudio de Temuco (18) muestra que un $87,2 \%$ de las embarazadas en el caso del calcio, un $89,4 \%$ para hierro y un $66 \%$ en el caso del zinc no alcanzan a cubrir un $75 \%$ de su requerimiento. El caso de Nigeria (17) muestra que las mujeres estudiadas presentaban una adecuación de $50 \%$ para calcio, $33 \%$ para hierro, $25 \%$ para cobre y $75 \%$ para el zinc. En el estudio de Finlandia (18) se señala que la adecuación del consumo de hierro es de un $42 \%$. Los países latinoamericanos muestran en el caso de Perú (20) que el consumo de calcio solo cubre un $20 \%$ de los requerimientos, $7 \%$ para hierro y $16 \%$ del zinc necesario en un día. En Venezuela (15) se muestra una adecuación para el consumo de calcio de $45 \%$ y $65 \%$ para hierro.

Un antecedente poco profundizado en los estudios realizados en embarazadas es el consumo de alimentos. El grupo estudiado mostró un consumo de alimentos con predominancia de los cereales, lo que es habitual en los países de Latinoamérica $(9,10)$. Al comparar la cantidad de porciones de los diferentes grupos de alimentos recomendadas a consumir por día para este grupo (26), evidenciamos importantes déficit en el consumo de la mayoría de los grupos de alimentos, es así como la adecuación del consumo de verduras está alrededor del 50\%, al igual que el consumo del grupo de las carnes, por otra parte el consumo de lácteos y de frutas es de $60 \%$ de lo recomendado, el grupo de aceites y grasas es consumido en un $80 \%$ de la recomendación y por el contrario el consumo del grupo de cereales supera largamente la recomendación para este grupo, sin presentar grandes diferencias entre los dos períodos estudiados. Investigaciones realizadas en el extranjero como la de España (19), muestra un bajo consumo de cereales, lácteos y verduras pero un consumo adecuado de frutas. El único estudio con antecedentes sobre consumo de alimentos en embarazadas en nuestro país es el de Concepción (13), que muestra un inadecuado consumo de lácteos y verduras, coincidiendo con los antecedentes obtenidos

\section{TABLA 7}

Comparación de ingestas a la semana 20 y semana 35 de gestación $(n=411)^{*}$

\begin{tabular}{lccc} 
& Semana 20 & Semana 35 & p \\
\hline Energía (Kcal./día) & $2267 \pm 696$ & $2202 \pm 696$ & $\mathrm{~ns}$ \\
Proteínas (g/día) & $82,5 \pm 28,5$ & $77,9 \pm 26,5$ & 0,005 \\
Vitamina A ( $\mu$ g/día) & $971,0 \pm 1637,9$ & $940,5 \pm 1420,9$ & $\mathrm{~ns}$ \\
Vitamina C (mg/día) & $115,7 \pm 75,4$ & $116,0 \pm 92,7$ & $\mathrm{~ns}$ \\
Vitamina E (mg/día) & $20,5 \pm 10,9$ & $20,8 \pm 11,5$ & $\mathrm{~ns}$ \\
Vitamina B6 (mg/día) & $1,7 \pm 0,8$ & $1,6 \pm 0,8$ & $\mathrm{~ns}$ \\
Calcio (mg/día) & $933,1 \pm 420,0$ & $887,4 \pm 389,9$ & $\mathrm{~ns}$ \\
Hierro (mg/día) & $19,6 \pm 7,7$ & $19,6 \pm 11,6$ & $\mathrm{~ns}$ \\
Magnesio (mg/día) & $272,3 \pm 110,5$ & $254,4 \pm 92,7$ & 0,005 \\
Zinc (mg/día) & $10,8 \pm 4,0$ & $10,1 \pm 3,8$ & 007 \\
\hline * t Student para muestras pareadas & & &
\end{tabular}


en este grupo de estudio.

La comparación de las ingestas de energía, proteínas y micronutrientes en los dos períodos del embarazo evaluados en este estudio, mostró solo diferencias significativas en la ingesta de proteínas, magnesio y zinc, siendo mayores en la semana 20 que en la semana 35. Es probable que por tratarse de embarazadas que presentan un déficit de peso al inicio, reciban con mucha atención las recomendaciones entregadas sobre la alimentación, tendientes a mejorar su estado nutricional al inicio del embarazo, con lo que su consumo de alimentos es levemente mayor que en el resto de la gestación.

Existen numerosas experiencias realizadas en el mundo y también en nuestro país con el objetivo de poder corregir los déficit de micronutrientes en embarazadas. Un ejemplo es la fortificación de la harina de panificación con ácido fólico en nuestro país en el año 2000, con ella se observó que la tasa de nacimientos de niños con defectos del tubo neural cayó un $40 \%$ entre los años 2000 y 2002 (47).

Con estos antecedentes, parece ser que la fortificación de alimentos y la suplementación con micronutrientes a las embarazadas, se logran mejorar las complicaciones derivadas de la mala ingesta de este grupo de la población.

\section{RESUMEN}

El objetivo del estudio es conocer la ingesta de nutrientes y alimentos de las embarazadas bajo peso de la Región Metropolitana Sur-Oriente. Se estudiaron 411 embarazadas que asistían a control de salud durante los años 2002 al 2004. Se aplicó una encuesta por recordatorio de 24 horas domiciliarias en la semana 20 y 35 por Nutricionistas. Las dietas fueron analizadas utilizando el software Food Processor 7,9, posteriormente se comparó el consumo con las porciones recomendadas por el Ministerio de Salud. Características basales (promedio y D.S.): edad, 23,8 \pm 5,4 años; peso, 49,3 $\pm 4,7 \mathrm{~kg}$; talla, $156,9 \pm 5,9 \mathrm{~cm}$; edad gestacional, $11,5 \pm 3,7$ semanas. $\mathrm{Al}$ comparar la ingesta con las recomendaciones se encontró un consumo adecuado de energía y macronutrientes, se encontraron valores menores del $75 \%$ de la ingesta recomendada para vitamina $\mathrm{A}, \mathrm{C}, \mathrm{E}$ y $\mathrm{B} 6$, calcio y zinc. La ingesta de ácidos grasos omega-3 fue en promedio de $61 \pm 50$ y $71 \pm 24 \mathrm{mg}$ /día en la semana 20 y 35 , un sexto de la recomendación NIH. Al comparar la ingesta de alimentos con las recomendaciones, se encontraron ingestas deficientes para, lácteos, frutas, verduras y carnes, por el contrario se encontró una ingesta superior de cereales. Conclusiones: La ingesta de nutrientes en este grupo de embarazadas es insuficiente en varios micronutrientes, producto del inadecuado consumo de alimentos, ello debiera ser corregido, mediante la mejoría de la calidad de su alimentación u otras acciones tales como la suplementación de estos micronutrientes en alimentos de consumo frecuente.

Palabras clave: alimentación, embarazo, estado nutricional.

Dirigir la correspondencia a:

Nut. Oscar Castillo Valenzuela

Departamento de Nutrición, Diabetes

y Metabolismo

Facultad de Medicina

Pontificia Universidad Católica de Chile

Lira 40, edificio de Gastroenterología, $4^{\circ}$ piso

Santiago.

Fax: (56-2) 6338298

oscarc@med.puc.cl

Agradecimientos: Este estudio fue financiado por Parmalat SpA, Italia, quien proporcionó el producto Mamán.

\section{BIBLIOGRAFÍA}

1. United Nations Administrative Committee on Coordination. Sub-Committee on Nutrition. Nutrition Policy Paper No. 18, 2000. Sitio web de Naciones Unidas.

2. Rosso P. Nutrition and metabolism in pregnancy. New York: Oxford University Press, 1990.

3. Institute of Medicine, National Academy of Sciences. Nutrition during pregnancy. Washington, D.C.: National Academy Press, 1990.

4. Willet W. Overview of nutritional epidemiology. En "Nutritional Epidemiology". Willet W, editor. Oxford, EEUU: Oxford University Press; 1990. p. 3 - 19.

5. Binham S, Nelson M. Assessment of food consumption and nutrient intake. En: Marggets M, Nelson M., editors. Oxford, EEUU: Oxford University Press; 1991. p. 151-91.

6. Mardones F, Urrutia M-T, Villarroel L, Rioseco A, Castillo O, Rozowski J, Tapia JL, Bastías G, Bacallao J, Rojas I. Effects of a dairy product fortified with multiple micronutrients and omega-3 fatty acids on birth weight and gestation duration in Chilean pregnant women. Public Health Nutr 2008; 11 (1): $30-40$.

7. Mardones F, Rosso P. Diseño de una curva patrón de incrementos ponderales para la embarazada. Rev Med Chile 1997; 125: 1437-48.

8. Jury G, Urteaga C, Taibo M. Porciones de intercambio y composición química de los alimentos 
de la pirámide alimentaria chilena. INTA, Centro de Nutrición Humana, Facultad de Medicina, Universidad de Chile. 1997.

9. Castillo O, Rozowski J, Cuevas A, Maiz A, Soto M, Mardones F, Leighton F. Ingesta de nutrientes en adultos mayores de la comuna de providencia, Santiago de Chile. Rev Med Chil 2002; 130:1335-42.

10. Castillo O, Rozowski J, Muñoz X, Cuevas A, Maiz A, Urquiaga I, Leighton F. "Ingesta de nutrientes en vegetarianos chilenos". Rev Chil Nutr 1998; 25:39-44

11. Mezzano D, Leighton F, Martínez C, Marshall G, Cuevas A, Castillo O, Panes O, Muñoz B, Pérez D, Mizón C, Rozowski J, San Martín A, Pereira J. "Complementary effects of mediterranean diet and moderate red wine intake on haemostatic cardiovascular risk factors". Eur J Clin Nutr 2001; 55: 444-51.

12. Simopoulos A. The mediterranean diets: what is so special about the diet of Greece? The scientific evidence. J Nutr 2001; 131: 3065S-73S.

13. Durán E, Soto D, Asenjo G, Pradenas F, Quiroz V. Evaluación de la dieta de embarazadas de área urbana y su relación con el estado nutricional. Rev Chil Nutr 1999; 26: 62-9.

14. Díaz C, Valeria O, Biolley E. Ingesta dietaria de nutrientes críticos en embarazadas. Rev Chil Nutr 2005; 32: 225-31.

15. Peña E, Sánchez A, Portillo Z. Evaluación dietética de adolescentes embarazadas durante el primer, segundo y tercer trimestre. ALAN 2003; 53: 133-40.

16. Pobocik R, Benavente J, Boudreau N, Spore C. Pregnant adolescents in Guam consume diets low in calcium and other micronutrients. J Am Diet Assoc 2003; 103: 611-4.

17. Oguntona C, Akinyele I. Food and nutrient intakes by pregnant Nigerian adolescents during the third trimester. Nutrition 2002; 18: 673-679.

18. Erkkola M, Karppinen M, Järvinen A, Knip M, Virtanen S. Folate, vitamin D, and iron intakes are low among pregnant Finnish women. Eur J Clin Nutr 1998; 52: 742-8.

19. Irles J, Iglesias E, Avilés S, Bernal E, Benito del Valle P, Moriones L, Maetzu A, Mingo D. Valor nutricional de la dieta en embarazadas sanas. Resultados de una encuesta dietética en gestantes. Nutr Hosp 2003; 18: 248-52.

20. Sacco I, Caulfield I, Zavaleta N, Betamozo L. Dietary pattern and usual nutrient intakes of Peruvian women during pregnancy. Eur J Clin Nutr 2003; 57: 1492-7.

21. FAO/WHO/UNU. Expert consultation. Human energy requeriments. Rome. World Health Orga- nization. 2005.

22. Food and Nutrition Board. Dietary Reference Intakes for calcium, Phosphorus, Magnesium, Vitamin D and Fluoride. National Academy Press, Washington D.C. 1997.

23. Food and Nutrition Board. Dietary Reference Intakes: Thiamin, Riboflavin, Niacin, Vitamin B6, Folate, Vitamin B12, Pantothenic Acid, Biotin and Choline. National Academy Press, Washington D.C. 1998.

24. Food and Nutrition Board. Dietary Reference Intakes: Vitamin A, Vitamin K, Arsenic, Boron, Chromium, Copper, Iodine, Iron, Manganese, Molybdenum, Nickel, Silicon, Vanadium and Zinc. National Academy Press, Washington D.C 2001.

25. Food and Nutrition Board. Dietary Reference Intakes: Vitamin C, Vitamin E, Selenium and Carotenoids. National Academy Press, Washington D.C 2000.

26. Uauy R, Atalah E, Barrera C, Behnke E. Alimentación y nutrición durante el embarazo. En Guías de alimentación para la mujer. Burrows R, Castillo C, editores. Universidad de Chile. INTA; Ministerio de Salud 2001.

27. ICNND. Nutrition Survey, Chile, March - June 1960. A report by the Interdepartmental Committee of nutrition for National Defense. Agosto 1961.

28. Ministerio de Salud. Encuesta continuada sobre el estado nutricional de la población chilena (ECEN). Julio 1974 - Junio 1975. Santiago de Chile 1976.

29. Castillo C, Atalah E, Benavides X, Urteaga C. Patrones alimentarios en adultos que asisten a consultorios de atención primaria en la región metropolitana. Rev Méd Chil 1997; 125: 283-9.

30. Butte N, Wong W, Treuth M, Ellis K, O'Brian E. Energy requirements during pregnancy based on total energy expenditure and energy deposition. Am J Clin Nutr 2004; 79: 1078-87.

31. Costello A, Osrin D. Micronutrient status during pregnancy and outcomes for newborn infants in developing countries. J. Nutr. 2003; 133: 1757S-64S.

32. Prentice A. Micronutrients and the bone mineral content of the mother, fetus and newborn. J Nutr 2003; 133: 1693S-9S.

33. Scholl T, Johnson W. Folic Acid: influence on the outcome of pregnancy. Am J Clin Nutr 2000; 71: 1295S-303S.

34. Gambling L, Danzeisen R, Fosset C, Andersen H, Dunford S, Srai K, Mcardle H. Iron and copper interactions in development and the effect on pregnancy outcome. J Nutr 2003; 133: 1554S-6S.

35. King J. Determinant of maternal zinc status during 
pregnancy. Am J Clin Nutr 2000; 71: 1334S-43S.

36. Goldenberg R. The plausibility of micronutrient deficiency in relationship to perinatal infection. $\mathrm{J}$ Nutr 2003; 133: 1645S-48S.

37. Azaïs-Braesco V, Pascal G. Vitamin A in pregnancy: requeriments and safety limits. Am J Clin Nutr 2000; 71: 1325S-33S.

38. Hollis B, Wagner C. Assesment of dietary Vitamin $\mathrm{D}$ requeriments during pregnancy and lactation. Am J Clin Nutr 2004; 79: 717-26.

39. Fall C, Yajnik C, Rao S, Davies A, Brown N, Farrant J. Micronutrient and fetal growth. J Nutr 2003; 133: 1747S-56S.

40. Allen L. Multiple micronutrients in pregnancy and lactation: an overview. Am J Clin Nutr 2005; 81: 1206S-12S.

41. Keen C, Clegg M, Hanna L, Lanque L, Rofers J, Daston G, Oteíza P, Uriu-Adams J. The plausibility of micronutrient deficiencies being a significant contributing factor to the occurrence of pregnancy complications. J Nutr 2003; 133: 1597S-605S.
42. Roberts J, Balk J, Bodnar L, Belizán J, Bergel E, Martínez A. Nutrient involvement in preeclampsia. J Nutr 2003; 133: 1684S-92S.

43. Mathews F, Youngman L, Neil A. Maternal circulating nutrient concentrations in pregnancy: implications for birth and placental weights of term infants. Am J Clin Nutr 2004; 79: 103-10.

44. Otto S, Van Houwelingen A, Badart-Smook A, Hornstra G. Changes in maternal essential fatty acid profile during early pregnancy and the relation of the profile to diet. Am J Clin Nutr 2001; 73: 302-7.

45. Emil S, Refsum H, Irgens L, Mork B, Tverdal A, Gjessing H, Bjorke A, Magne P. Plasma total homocysteine, pregnancy complications and adverse pregnancy outcomes: the Hordaland Homocysteine Study. Am J Clin Nutr 2000; 71: 962-8.

46. Tamura T, Goldenberg R, Johnston K, Dubard M. Maternal plasma zinc concentrations and pregnancy outcome. Am J Clin Nutr 2000; 71: 109-13.

47. Hertrampf E, Cortés F. Folic acid fortification of wheat flour: Chile. Nutr Rev 2004; 62: 44S-8S. 